\title{
Magnetic Induction Tomography: Receiver Circuit and Its Design Criteria
}

\author{
Zulkarnay Zakariaa,b*, Muhammad Saiful Badri Mansor ${ }^{b}$, Ruzairi Abdul Rahim ${ }^{b}$, Ibrahim Balkhis ${ }^{a}$, Mohd Hafiz Fazalul Rahimana, Nor \\ Muzakkir Nor Ayob ${ }^{b}$, Herlina Abdul Rahimb ${ }^{b}$, Sazali Yaacoba \\ ${ }^{a}$ Tomography Imaging Research Group, School of Mechatronic Engineering, Universiti Malaysia Perlis, 02600, Arau, Perlis, Malaysia \\ ${ }^{b}$ Process Tomography and Instrumentation Engineering Research Group (PROTOM-i), Infocomm Research Alliance, Faculty of Electrical Engineering, \\ Universiti Teknologi Malaysia,UTM Skudai, Johor 81310, Malaysia \\ *Corresponding author: zulkarnay@unimap.edu.my
}

\section{Article history}

Received :23 April 2013

Received in revised form : 2 June 2013

Accepted :15 July 2013

Graphical abstract
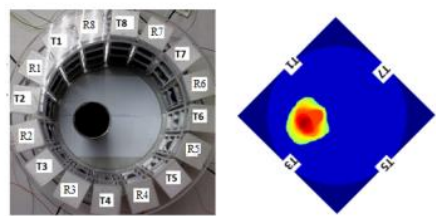

\section{Abstract}

This paper discusses the receiver circuit and criteria for component selection towards the application of a real-time magnetic induction tomography system. Component selection plays an important role since image reconstruction of the object of interest with high quality and at a higher frame rate cannot be achieved without the right parameter criteria. The demands for a high quality imaging system have recently been increasing, especially in industrial processes involving dynamic movement, thus this paper may provide valuable information on better magnetic induction tomography system implementation for industrial processes and biomedical imaging through the use of a coil as a transmitter and also a receiver. The linear back projection algorithm has been employed in this system and has proven capable of identifying the location and size of the object based on the reconstructed images.

Keywords: Magnetic induction tomography; frame rate; components selection; industrial process; biomedical

\subsection{INTRODUCTION}

A magnetic induction tomography (MIT) system is categorized as part of the passive imaging family together with electrical impedance tomography (EIT), electrical capacitance tomography (ECT) and magnetostatic permeability tomography (MPT) [1]. As represented by the family name, MIT is sensitive to all three passive electrical properties (PEPs) namely conductivity $(\sigma)$, permittivity $(\varepsilon)$ and permeability $(\mu)$; however, in most cases, either in industrial or biomedical applications, conductivity has become the choice due to its dominance in comparison to permittivity and permeability, especially within the range of the $\beta$-dispersion region $(10 \mathrm{kHz}-10 \mathrm{MHz})$ for biological tissue imaging [2][3]. The electrode less and contactless schemes of MIT modality have become of interest to the researchers because errors due to ill-defined electrode-skin contact and electrode positioning can be avoided through this technique [4] while at the same time providing more freedom for object movement.

The architecture of an MIT system consists of excitation coils, receiving coils (sensors), sensing electronics and a PC or micro-controller. A PC or micro-controller communicates with the sensing electronics circuits in controlling the excitation coil signal projections, while at the same time collecting data at the receiver. A PC also locates the image reconstruction algorithm where the measured data from the sensing electronics is processed and utilized to reconstruct the images of the objects $[5,16]$. The general MIT architecture is shown in Figure 1.

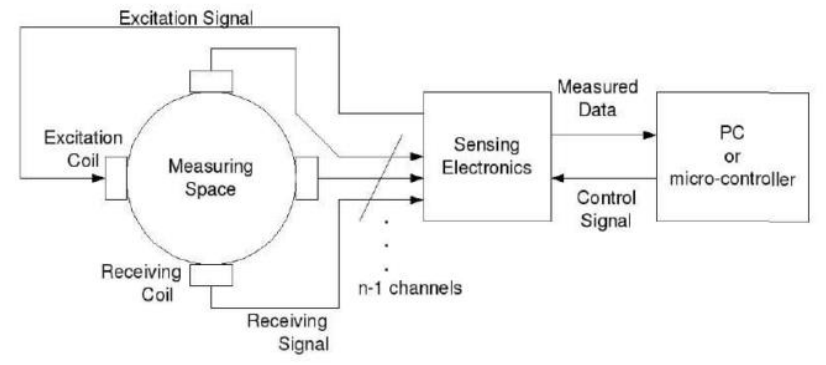

Figure 1 MIT system architecture [5]

The principle of MIT modality is the application of an electromagnetic field, generated by the excitation coils, known as the primary field, which then propagates and penetrates objects located within the imaging space or region of interest (ROI). Due to the PEPs of the object, an eddy current is induced inside the object itself, hence this eddy current then produces its own field, known as the secondary field or eddy current field [6]. The PEPs of the material are represented by the magnitude and phase of the generated secondary field, thus it becomes the only interested field to be detected at the receiver.

The nature of the physics phenomenon existing in the MIT measuring space follows the famous governed Maxwell equation 
[7] which includes Faraday's law, Ampere's law and Coloumb's Law.

\subsection{MIT ISSUES}

The objective of any tomography system is to produce images which provide important information on the object of interest. The challenges in biological tissue imaging, with most of the applications being of the static imaging type, are high resolution and high accuracy output [8], whereas in industrial processes, where, normally, dynamic imaging is involved, high speed processing is crucial. In a dynamic imaging system with an adopted real-time scheme, images at a higher frame rate are generated to handle very fast changes in the object properties [9]. Other issues that need to be overcome are the associated nonlinear and ill-posed problems which exist in an MIT system [10]. Since these issues are directly related to the data achieved from the receiver circuit, extra attention needs to be given to the development of the receiver circuit itself. This paper discusses the component selection criteria of the receiver circuit in reducing the potential for or sources of errors and the processing time for the data processing before reviewing the image reconstruction algorithm.

\subsection{MIT RECEIVER CIRCUIT}

The receiver section consists of a sensor/receiver coil, a signal conditioning circuit and a micro-controller. A signal detected at the receiver coil is then transferred to the signal conditioning circuit for processing. At this stage, the signal has to undergo five processing stages; pre-amplifier, amplifier, peak detector, isolation amplifier and buffer, as shown in Figure 2, before being passed to a micro-controller/computer for image reconstruction.

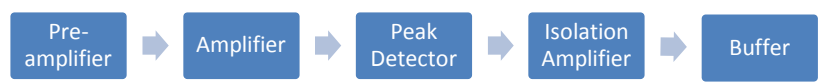

Figure 2 Block diagram of the signal conditioning circuit at the receiver side

\subsection{Pre-amplifier}

A pre-amplifier forms the first stage of the received signal that the receiver coil will reach. The received signal at the receiver coil is very low, in the $\mathrm{mV}$ range. For this purpose, the instrumentation amplifier INA111, as shown in Figure 3 was selected. INA111 has the capability of amplifying the $\mathrm{mV}$ range of the signal besides having high speed, a high slew rate and low noise. This FET-input instrumentation amplifier also offers excellent performance due to the current-feedback topology inside, which provides extended bandwidth $(2 \mathrm{MHz}$ at Gain $=10)$ and a fast settling time ( 4 us at Gain $=100$ ). The range of the gain varied from 1 to over 1000 and can be set by a single external resistor [11].

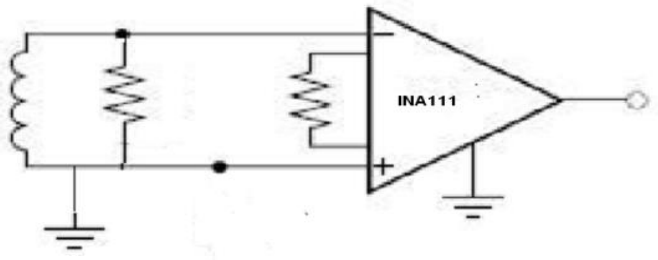

Figure 3 Pre-amplifier circuit using INA111

The input impedance of the INA111 is extremely high, approximately $10^{12} \Omega$. However, a path must be provided for the input bias current of both inputs. This input bias current is typically less than $10 \mathrm{pA}$. High input impedance means that this input bias current will change little with the varying input voltage.

\subsection{Amplifier}

The AD817 op amp was chosen for the amplifier. The AD817 is low cost, low power, can operate on single or dual supply and is a high speed op amp. A non-inverting amplifier configuration was used, as shown in Figure 4.

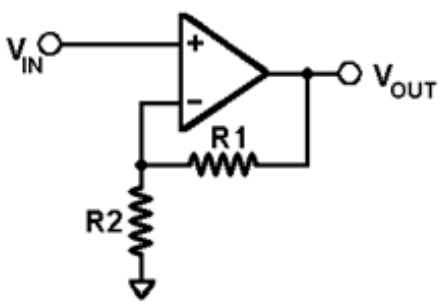

Figure 4 Amplifier circuit using AD817

The advantage of non-inverting is that it offers higher input impedance than the inverting amplifier circuit. The gain, $A_{v}$, of a non-inverting amplifier can be determined by Equation (1).

$$
A_{v}=1+\frac{R_{1}}{R_{2}}
$$

The AD817 op amp was chosen as the amplifier due to its high speed with the $50 \mathrm{MHz}$ unity gain bandwidth, $350 \mathrm{~V} / \mathrm{us}$ slew rate and a settling time of $45 \mathrm{~ns}(0.1 \%)$. Furthermore, it has a low noise characteristic [12].

\subsection{Peak Detector}

Due to its high speed characteristic, the AD817 was implemented in building a fast peak detector. The peak detector, as shown in Figure 5, is used to hold the maximum value of the input signal before the signal is measured by applying a capacitor to store the peak voltage value. When the input voltage increases, the capacitor is charged up to the new hold peak value, and if the input voltage is reduced, the diode will keep the capacitor from being discharged. The IN4148 diode was chosen for its high speed of $4 \mathrm{~ns}$ reverse recovery time [13].The second op amp is only used as a buffer. 


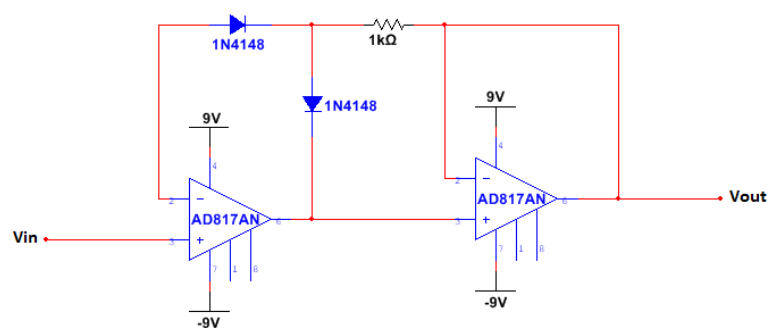

Figure 5 Circuit diagram of the peak detector

\subsection{Isolation Amplifier}

An isolation amplifier, as shown in Figure 6, protects dataacquisition components from the potentially destructive voltages present at remote transducers. These amplifiers are also useful when amplifying low-level signals in multi-channel applications. They can also eliminate measurement errors caused by ground loops. The ISO124 was chosen for this project due to its precision lowest-cost isolation amplifier incorporating a novel duty cycle modulation-demodulation technique. In ISO124, the signal is transmitted digitally across a $2 \mathrm{pF}$ differential capacitive barrier. The barrier characteristics do not affect signal integrity due to digital modulation, resulting in excellent reliability and good high-frequency transient immunity across the barrier. Both barrier capacitors are imbedded in the plastic body of the package. The non-linearity of the ISO124 is very low, that is, $0.01 \%$ [14].

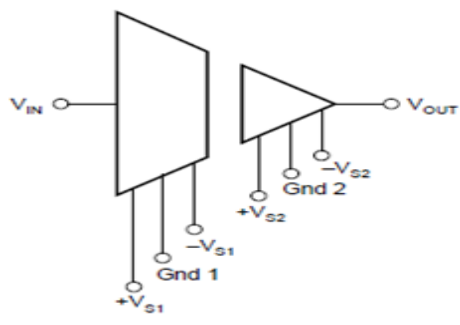

Figure 6 Internal structure of the isolation amplifier

\subsection{Buffer}

A buffer is used to avoid signal loss when connecting to a circuit with low input impedance. The HA5002 buffer was chosen for this project. The HA5002, as shown in Figure 7, has high input impedance $(300 \mathrm{k} \Omega)$ with low output impedance $(3 \Omega)$. The HA5002 buffer can be used for high speed due to its very wide bandwidth (110 MHz) and very high slew rate (1300 V/us) [15].

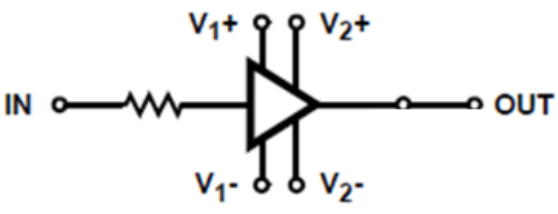

Figure 7 Internal structure of the HA5002 buffer

\subsection{EXPERIMENTAL SET-UP}

In the developed MIT system, there are eight excitation coils and eight receiver coils arranged alternately in a circle with a $26.5 \mathrm{~cm}$ diameter ROI, as shown in Figure 8. An input current of 140 $\mathrm{mA}_{\mathrm{pp}}$ at a frequency of $500 \mathrm{kHz}$ was fed into the excitation coil with an empty ROI (no objects) and the data sample rate was 1 sample for 10 minutes which finally produced 600 data items in total in each receiver channel, as shown in Figure 9. Room temperature was set to $25{ }^{\circ} \mathrm{C}$ to avoid the effects of temperature change on the measurements. Evaluations of the developed hardware consisted of assessing the sensitivity of each receiver, the stability of the system and the quality of the reconstructed image. For the evaluation of the sensitivity of each receiver and the system's stability only Tx1 was activated, while all the receivers were ready for data collection. On the other hand, for evaluation of the reconstructed images, the complete MIT system was activated.

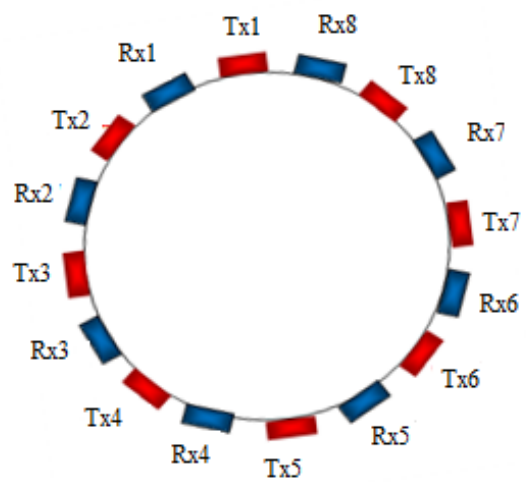

Figure 8 Excitation coil (Tx) and receiver (Rx) arrangement of the developed MIT system

\subsection{RESULTS}

\subsection{Sensitivity of Receivers}

The sensitivity of each receiver should be mirrored to each other as the activated excitation coil becomes the centre line. In this experiment, as Tx1 was activated, all the receivers produced almost the same value as the receiver mirrored to them, as shown in Figure 9.

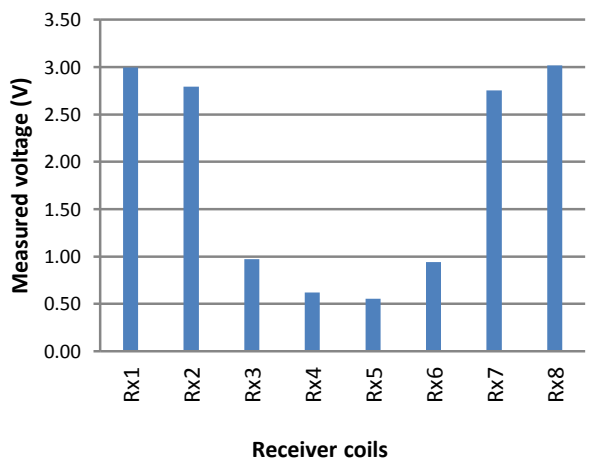

Figure 9 Sensitivity of the receiver coils when Tx1 transmits 
The pairs were Rx1-Rx8 ( $\pm 3.00 \mathrm{~V}), \mathrm{Rx} 2-\mathrm{Rx} 7( \pm 2.80 \mathrm{~V}), \mathrm{Rx} 3-\mathrm{Rx} 6$ $( \pm 0.90 \mathrm{~V})$ and $\mathrm{Rx} 4-\mathrm{R} \times 5( \pm 0.60 \mathrm{~V})$. Significantly higher values can be observed for the receivers located at either side of the activated $\mathrm{Tx}$ and quite smaller values for the receivers further away from the Tx. The difference in the reduction between each pair is non-linear due to the nature of the electromagnetic field itself, which is also non-linear. The slight difference in value in a pair is due to the small difference in the inductance of the hand wound receiver coil which is within the tolerance of $\pm 5 \mathrm{mH}$.

\subsection{Stability of the System}

Stability testing is crucial in analyzing the appropriate warming up time for the system to become stable before measurements are taken. The dynamic range of the signals for all the channels proved that the system is very stable from the start of the experiment, as shown in Figure 10, and the standard deviation for each receiver channel is as in Table 1. The standard deviation of each receiver channel is very small and consistent, representing the stability of the measured signals.

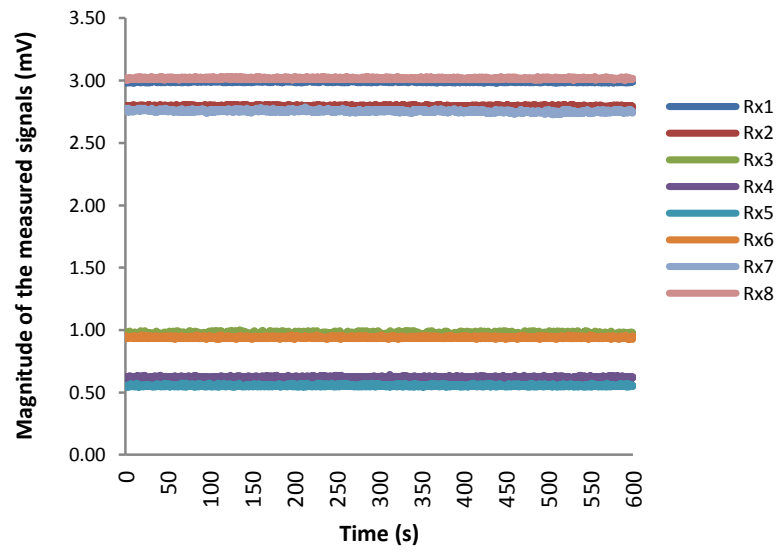

Figure 10 Dynamic range of all receiver coils

Table 1 Standard deviation of measured data of each receiver

\begin{tabular}{cc}
\hline Receiver & Standard Deviation, $\boldsymbol{\sigma}$ \\
\hline $\mathrm{Rx} 1$ & 0.0056 \\
$\mathrm{R} 2$ & 0.0048 \\
$\mathrm{R} \times 3$ & 0.0074 \\
$\mathrm{R} 44$ & 0.0046 \\
$\mathrm{R} 5$ & 0.0057 \\
$\mathrm{R}$ 6 & 0.0057 \\
$\mathrm{R} 7$ & 0.0085 \\
$\mathrm{Rx} 8$ & 0.0050 \\
\hline
\end{tabular}

\subsection{Reconstructed Image}

The reconstructed image of the object of interest shows $94.3 \%$ accuracy with respect to the position based on its centre, but not on the size and shape of the object, as shown in Figure 11. The object is hollow and round in shape; however, the image is solid and almost square. Higher intensity colour at the centre region of the image is due to the sum of the projected values from each receiver, which in turn contributed to the solid image. The accuracy of size, position and shape can be improved with some modifications or changes to the image reconstruction algorithm, since the linear back projection (LBP) algorithm currently being used is the basic algorithm in tomography. LBP works well with the linear system but produced artefacts in the image when implemented in a nonlinear system. This shows that LBP is insufficiently capable of being implemented in a non-linear system. Thus, a non-linear image reconstruction algorithm may provide the best solution to this problem.
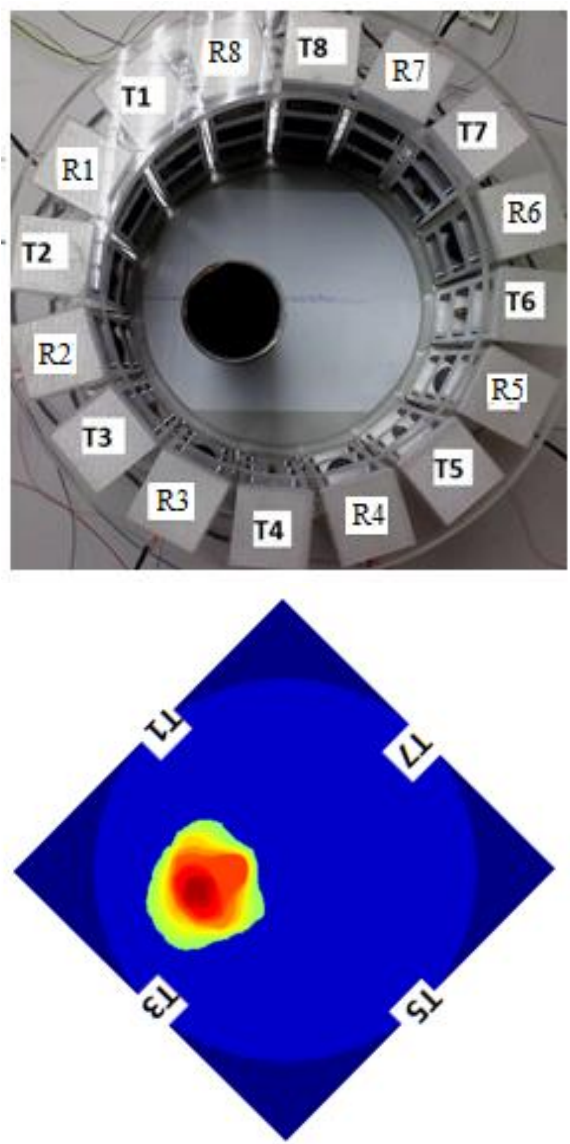

Figure 11 Reconstructed images of a steel pipe sample

\subsection{CONCLUSIONS}

This paper presents the design criteria for an MIT receiver circuit in developing a sensitive and stable system to produce quality reconstructed images of the objects. Through these criteria, the evaluations have proved that the developed MIT system has the capability to be a complement to the current imaging system, but with certain modifications and upgrading of the LBP image reconstruction algorithm. More work has to be carried out to test the effectiveness of the system.

\section{Acknowledgement}

The authors would like to thank the Universiti Teknologi Malaysia, Universiti Malaysia Perlis and Ministry of Science Technology and Innovation for supporting this research. This work is supported by Science Fund Grant 06-01-06-SF0889. 


\section{References}

[1] M. Soleimani. 2008. Computational Aspects of Low Frequency Electrical and Electromagnetic Tomography: A Review Study. International Journal of Numerical Analysis and Modelling. 5(3): 407-440.

[2] H. Griffiths. 2001. Magnetic Induction Tomography. Measurement Science and Technology. 12(2): 1126-1131.

[3] Z. Zakaria, R. Abdul Rahim, M. S. B. Mansor, S. Yaacob, N. M. Nor Ayob, S. Z. Mohd Muji, and M. H. Fazalul Rahiman. 2012 Advancements in Transmitters and Sensors for Biological Tissue Imaging in Magnetic Induction Tomography. Sensors. 12(6): 7126-7156.

[4] R. Merwa and H. Scharfetter. 2007. Magnetic Induction Tomography: Evaluation of the Point Spread Function and Analysis of Resolution and Image Distortion. Physiological Measurement. 28(7): S313-24.

[5] L. Ma, H.-Y. Wei, and M. Soleimani. 2012. Pipelines Inspection Using Magnetic Induction Tomography Based on a Narrowband Pass Filtering Method," Progress In Electromagnetics Research. 23: 65-78.

[6] W. Yin, G. Chen, L. Chen, and B. Wang. 2011. The Design of a Digita Magnetic Induction Tomography ( MIT ) System for Metallic Object Imaging Based on Half Cycle Demodulation. IEEE Sensors Journal. 11(10): 2233-2240

[7] R. Casanova, A. Silva, and A. R. Borges. 2004. A Quantitative Algorithm for Parameter Estimation in Magnetic Induction Tomography. Measurement Science and Technology. 15(7): 1412-1419.

[8] Y. Maimaitijiang, H. C. Wee, A. Roula, S. Watson, R. Patz, and R. J. Williams. 2009. Evaluation of Parallel FFT Implementations on GPU and
Multi-core PCs for Magnetic Induction Tomography. n WC 2009, IFMBE Proceedings 25/IV. 1889-1892.

[9] Y. Granot, A. Ivorra, and B. Rubinsky. 2007. Frequency-division Multiplexing for Electrical Impedance Tomography in Biomedical Applications. International Journal of Biomedical Imaging. 1-9.

[10] B. Dekdouk, W. Yin, C. Ktistis, D. W. Armitage, and A. J. Peyton. 2010. A Method to Solve the Forward Problem in Magnetic Induction Tomography Based on the Weakly Coupled Field Approximation. IEEE Transactions on Biomedical Engineering. 57(4): 914-21.

[11] Burr-Brown. 1998. INA 111-High Speed FET-Input Instrumentation Amplifier. Data Sheet. 1-16.

[12] Analog Devices. 1995. AD817-High Speed, low Power Wide Supply Range Amplifier. Data Sheet. 1-7.

[13] NXP Semiconductors. 2004. 1N4148-High Speed Diodes. Product Data Sheet. 1-9.

[14] Texas Instruments. 2005. ISO124-Precision Lowest-Cost Isolation Amplifier. Data Sheet. 1-18.

[15] Intersil. 2006. HA5002-Monolithic, Wideband, High Slew Rate, High Output Current Buffer. Data Sheet. 1-7.

[16] R. Abdul Rahim. S.Z Mohd. Muji. January 2013. Optical Tomography: Image Improvement using Mix Projection of Parallel and Fan Beam Mode. Measurement Journal (ISSN: 0263-2241) Elsevier Science. 46: 1970 - 1978 